\title{
Biosorption of Heavy Metals
}

\author{
Saba Shamim \\ Additional information is available at the end of the chapter
}

http://dx.doi.org/10.5772/intechopen.72099

\begin{abstract}
Industrialization has led to introduction of heavy metals in the environment. Heavy metals are known to persist in the environment and become a risk for organisms. Microorganisms are present in industrial effluents. They have adopted different strategies to cope up with the harmful effects of these metals. These strategies can be metabolism dependent or independent. One such strategy is biosorption which is binding of metal ions with metal binding proteins present on the cell wall. Biosorption is exhibited by bacteria, algae, fungi and yeasts. Not only living organisms, but also residuals of dead bodies of microorganisms shows biosorbent properties like agricultural wastes including husk, seeds, peels and stalks of different crops. Different factors affect the rate of biosorption which includes temperature, $\mathrm{pH}$, nature of biosorbents, surface area to volume ratio, concentration of biomass, initial metal ion concentration and metal affinity to biosorbent. Various models including Freundlich model and Langmuir model can be used to describe biosorption. Recovery of biosorbed metals can be done using agents like thiosulfate, mineral acids and organic acids. Choice of desorption agent should be carefully selected to prevent alteration of physical properties of a biosorbent.
\end{abstract}

Keywords: biosorption, heavy metals, bacteria, algae, fungi, yeasts

\section{Introduction}

Nature has gifted our earth with four spheres; biosphere, lithosphere, hydrosphere, and atmosphere. Together these spheres are important for maintaining a balanced ecosystem [1]. The industrial revolution in the past five decades is remarkable. Due to anthropogenic activities, increasing population, industrialization and urbanization, all spheres have become polluted [2-7]. There are two main sources of introduction of heavy metals in the environment (1) natural sources which includes volcanic emissions, forest fires, deep-sea vents, and geysers [8] and (2) anthropogenic sources which includes mining and smelting sites, metalmanufacturing plants, painting- and coating-industries and tanneries. These heavy metals are 
released directly into the environment. Metals exhibit health issues [9] if their concentrations exceed allowable limits. Even when the concentration of metals does not exceed these limits, there is still a potential for bioaccumulation and associated chronic toxicity as heavy metals are known to be accumulative within biological systems [10]. These metals include arsenic, cadmium, chromium, copper, lead, mercury, nickel, and zinc [4, 11]. Industrial effluents are known to contain heavy metals which originate from metal plating, mining activities, smelting, battery manufacture, tanneries, petroleum refining, paint manufacture, pesticides, pigment manufacture, and printing and photographic industries [1, 11-14].

\section{Heavy metals}

Heavy metals are usually defined as metals having density more than $5 \mathrm{~g} / \mathrm{cm}^{3}$ [15]. They are classified as essential and non-essential metals. The metals which are need for normal cellular growth are essential metals e.g. zinc, nickel, copper, etc. Such metals are required in low concentrations (nM), but at higher concentrations ( $\mu \mathrm{M}$ to $\mathrm{mM}$ ) all heavy metals have detrimental effects to organisms $[16,17]$. If the metals have no known biological function, they are called as non-essential metals e.g. e.g., lead, cadmium, mercury [18]. Such metals are toxic at any concentration [8]. The list of essential and non-essential heavy metals is given (Table 1). There are 90 naturally occurring elements in periodic table, 21 are non-metals, 16 are light metals and the remaining 53 (with As included) are heavy metals [19]. In periodic table, transition elements are mostly heavy metals. They have incompletely filled ' $\mathrm{d}$ ' orbitals which allow heavy-metal cations to form complex compounds that may or may not be redox-active. In this way, heavy metals play an important role as 'trace elements' (cobalt, copper, nickel, and zinc) in sophisticated biochemical reactions and are important cofactors for metallo-proteins and enzymes [8]. The toxicity of heavy metal ions starts when their concentration becomes higher

\begin{tabular}{ll}
\hline Category of heavy metal & Example of heavy metals \\
\hline Essential & Copper $(\mathrm{Cu})$ \\
& Nickel $(\mathrm{Ni})$ \\
& Iron $(\mathrm{Fe})$ \\
& Zinc $(\mathrm{Zn})$ \\
& Magnesium $(\mathrm{Mg})$ \\
Non-essential & Lead (Pb) \\
& Mercury (Hg) \\
& Cadmium (Cd) \\
& Tin (Sn) \\
Arsenic (As)
\end{tabular}

Table 1. Essential and non-essential heavy metals. 
in the cells, due to which they form complex compounds [15, 18]. Microorganisms acquire resistance to these toxic metals by lateral gene transfer [20]. The interaction of microorganism with metal ions depends on factors like oxidation state of the metal ion, chemical/physical nature of metals, growth phase of microorganism etc. [21].

\section{Methods for removal of heavy metals}

Since last many decades, various physical and chemical methods were employed to remove metals from environment. The list is given below [5, 13, 14, 22-24].

Chemicals methods: Chemical precipitation, electrochemical treatment, oxidation/reduction.

Physical methods: Ion exchange, membrane technology, reverse osmosis, and evaporation recovery, filtration.

Biological methods: Microorganisms including bacteria, fungi or algae.

However, these strategies were not the first choice as they are expensive, inefficient, laborintensive, or the treatment process lacks selectivity $[25,26]$. The research on bioremediation or biosorption-based remediation techniques in the past decades has concluded that bioremediation is a natural process and cost effective [4, 27-31].

\section{Biosorption}

Biosorption is defined as "ability of biological materials to accumulate heavy metals from wastewater through metabolically mediated (by the use of ATP) or spontaneous physicochemical pathways of uptake (not at the cost of ATP), or as a property of certain types of inactive, non-living microbial biomass which bind and concentrate heavy metals from even very dilute aqueous solutions" $[1,5,32]$. It is a complex process that depends on differentfactors like cell physiology, physicochemical factors such as $\mathrm{pH}$, temperature, contact time, ionic strength, and metal concentration, chemistry of the metal ions, cell wall composition of microorganisms [5, 33, 34]. Biosorption of different heavy metals e.g. cadmium, silver, lead, nickel etc. by using microorganisms like fungi, algae or bacteria was studied by different groups [34-42].

\subsection{Significance}

Bioremediation offer different advantages such as low operating cost, minimum ratio of disposable sludge volume, high efficiency in detoxifying very dilute effluents and even in situ remediation $[30,43,44]$. Bacteria detoxify heavy metals in a variety of different ways [45]. Although various types of tolerance mechanisms have been reported in bacteria for heavy metal stress, $\mathrm{Cd}$ detoxification has only been restricted to efflux pumps. The plasmid encoded cad systems in (Staphylococcus aureus) and the czc system (Alcaligenes eutrophus) are 
best characterized. These systems actively maintain $\mathrm{Cd}$ ions outside the intracellular environment, hence avoiding its toxic effects. Consistently, many researchers reported that sensitive bacteria can accumulate 3-15 times more Cd than resistant strains [46]. The most important aspect of $\mathrm{Cd}$ ions is that they covalently bind to sulfhydryl groups. Although this is partially the cause for its high toxicity, this feature is also used by several organisms to render the metal harmless to the cell, through sequestration with metal-detoxifying ligands, the metal becomes less bioavailable.

\subsection{Advantages of biosorption}

Following are given the advantages of biosorption over conventional metal removal methods $[47,48]$.

1. Cheaper production of biomass (bacteria or fungi)

2. Use of biomass for removal of heavy metals

3. Multiple heavy metals uptake at a time

4. Treatment of large volumes of wastewater

5. No need for chemical additions as highly selective for uptake and removal of specific metals

6. Functional over wide range of conditions including temperature, $\mathrm{pH}$, presence of other metal ions, etc.

7. Easy and cheaper desorption of metals attached to biomass

8. Reduced volume of waste or toxic materials production

\subsection{Disadvantages of biosorption}

The disadvantages of biosorption are stated below [49].

1. Saturation of active sites of metal binding ligands

2. Reversible sorption of metals on biomass

\section{Biosorption mechanisms}

The process of heavy metal ion binding to bacterial cell wall (peptidoglycan) can be metabolism dependent or independent [1].

\subsection{Metabolism dependent biosorption}

Metabolism dependent biosorption is exhibited by living biological material. It involves various mechanisms like chelation; a specific way in which ions and molecules bind to metal ions 
and it involves the formation or presence of two or more separate coordinate bonds between a polydentate ligand and a single central atom, physical adsorption; adhesion of atoms, ions, or molecules from a gas, liquid, or dissolved solid to a surface. This process creates a film of the adsorbate on the surface of the adsorbent. It is a surface phenomenon. Generally the adsorption process is classified as physisorption, characteristic of weak van der Waals forces, or chemisorption, characteristic of covalent bonding. It may also occur due to electrostatic attraction, precipitation; it is the creation of a solid in a solution or inside another solid during a chemical reaction or by diffusion in a solid. When this reaction occurs in a liquid solution, the solid formed is called the "precipitate" and the chemical that causes the solid to form is called the "precipitant") or complexation (it consists of a central atom or ion, which is usually metallic and is called the coordination centre, and a surrounding array of bound molecules or ions, that are known as ligands or complexing agents. Many metal-containing compounds, especially those of transition metals, are coordination complexes). There may involve a single process or combination of these processes $[50,51]$. If the metal binding to cell wall is metabolism dependent then it involves energy from ATP. The ligands present on the cell wall of biological material such as phosphoryl, carboxyl, carbonyl, sulfhydryl and hydroxyl groups immobilizes the metal ion [32] and then uptake occurs [5]. Other factors that effect the metal uptake by living biomass includes nature of heavy metals ions, conditions of the medium, cell wall composition, etc. [5]. The uptake process by living biomass involves adsorption to cell wall and entering into the cytoplasm $[29,31,52,53]$.

\subsection{Metabolism independent biosorption}

The metabolism independent process mostly occurs in biomass consisting of dead cells [54]. The adsorption process is the main key point behind such physicochemical biosorption mechanism. The adsorption process can be ionic interactions or physiochemical adsorption. Presence of anionic ligands on bacterial cell wall (carboxyl, amine, hydroxyl, phosphate, and sulfhydryl groups) also plays an important role in metal biosorption. Living biological mass is preferred over dead mass, because living cells have ability for continuous metal uptake, and self-replenishment $[27,29,31]$. Previously it is reported that adsorption is a rapid process while accumulation is slow and energy dependent [29, 31 52-53]. The fate of metal inside cell can be accumulation, detoxification and/or efflux depending on the nature of bacteria $[31,55,56]$. In past few decades, many groups worked on heavy metal resistant bacteria that can be used for bioremediation [27, 29, 31, 56-58]. Many workers reported that cells of bacteria of genera Alcaligenes and Pseudomonas can be used for bioremediation purpose [45].

\subsection{Metal accumulation}

In order to have the physiological effect on the growth of cells, heavy metals must enter the cell $[19,59,60]$. Metal uptake system in bacteria is grouped in two types; one is fast and unspecific, constitutively expressed and does not require ATP. They are usually driven only by the chemiosmotic gradient across the cytoplasmic membrane of bacteria. The second type of uptake system is highly specific, slow, inducible and dependent on ATP, in addition to the chemiosmotic gradient. They are only induced in times of need, starvation or a special metabolic situation [61]. 
As cell surface encounter metal ion, formation of a complex takes place, which is a pre-requisite for uptake of metals by the organism $[59,60]$. Once surface sorption takes place, the metal is transported into the periplasmic space of Gram-negative cells and transported further into the cytoplasm [60]. When cell encounters high concentration of any heavy metal, the heavy metal ion is transported into the cytoplasm, accumulated inside the cell due to one type of metal uptake which is fast, unspecific, constitutively expressed and does not require ATP [61]. The cations of heavy metals interact with physiological ions $\mathrm{Cd}^{2+}$ with $\mathrm{Zn}^{2+}$ or $\mathrm{Ca}^{2+}, \mathrm{Ni}^{2+}$ and $\mathrm{Co}^{2+}$ with $\mathrm{Fe}^{2+}, \mathrm{Zn}^{2+}$ with $\mathrm{Mg}^{2+}$ thus inhibit the function of respective physiological cations. This result in oxidative stress in the cell [1].

\section{Types of biosorbents}

Biosorbents can be classified as living or non- living organic materials. They are discussed below in detail.

\subsection{Living organic materials}

\subsubsection{Bacteria}

Among microorganisms, bacteria constitute of being the most abundant, versatile, most diverse creature on this planet earth $[48,62]$. They are basically classified on the basis of their morphology as rod, cocci or spirillum [48, 63]. A bacterium has relatively simple morphology consisting of cell wall, cell membrane, capsule, slime layer and internal structures mitochondria, Golgi apparatus, ribosomes, endoplasmic reticulum. Slime layer contains functional groups like carboxyl, amino, phosphate or sulfate for metals chelation [48, 62]. Cell wall in general, is responsible for surface binding sites and binding strength for different metal ions depending on different binding mechanisms. Various bacterial species e.g. Bacillus, Pseudomonas, Escherichia [48] exhibit biosorption property because of their small size and ability to grow in different environmental conditions [64-66].

Gram classification divides bacteria in two broad categories; Gram positive and Gram negative. Gram negative mostly constitute pathogens although pathogens are also reported in Gram positive. Gram positive bacteria are comprised of thick peptidoglycan layer connected by amino acid bridges, also known to contain polyalcohols and teichoic acids. Overall, Gram positive bacterial cell wall comprised of $90 \%$ peptidoglycan. Some teichoic acids are linked to lipids of lipid bilayer forming lipoteichoic acid. These lipoteichoic acids are linked to lipids of cytoplasmic membrane. They constitute linkage of peptidoglycan to cytoplasmic membrane. This results in cross linking of peptidoglycan forming a grid like structure. These teichoic acids are responsible for negative charge on cell wall due to presence of phosphodiester bonds between teichoic acid monomers [48]. On the other hand, Gram negative bacterial cell wall contains an additional outer membrane composed of phospholipids and lipopolysaccharides. Gram negative cell wall contains 10-20\% peptidoglycan. The negative charge on the Gram negative bacteria is due to lipopolysaccharides, teichoic acids, teichuronic acids. Extracellular polysaccharides also exhibit the property of metal binding. They are not present in all Gram negative bacteria. Moreover, those species that contain them, they can be easily removed by chemical washing or mechanical disruption $[49,67]$. 


\subsubsection{Bacterial biosorption}

Bacterial cell wall encountering the metal ion is the first component of biosorption. The metal ions get attached to the functional groups (amine, carboxyl, hydroxyl, phosphate, sulfate, amine) present on the cell wall $[49,67]$. The general metal uptake process involves binding of metal ions to reactive groups present on bacterial cell wall followed by internalization of metal ions inside cell [48]. More metal is uptaken by Gram positive bacteria due to presence of glycoproteins. Less metal uptake by Gram negative bacteria is observed due to phospholipids and LPS [68,69]. Biosorption of various metals by different bacteria is given in Table 2 .

\begin{tabular}{|c|c|c|c|c|c|c|c|c|c|}
\hline $\begin{array}{l}\text { Sr. } \\
\text { No. }\end{array}$ & Metals & Bacteria & $\begin{array}{l}\text { Temperature } \\
\left({ }^{\circ} \mathrm{C}\right)\end{array}$ & $\mathrm{pH}$ & Agitation & Time & $\begin{array}{l}\text { Wt } \\
(g / L)\end{array}$ & $\begin{array}{l}\mathrm{q}(\mathrm{mg} / \mathrm{g}) \text { or } \\
\% \text { removal }\end{array}$ & References \\
\hline \multirow[t]{3}{*}{1.} & Arsenic & Bacillus sp. KM02 & - & - & - & - & - & - & [108] \\
\hline & & Kocuria sp. & - & - & - & - & - & - & [109] \\
\hline & & Bacillus sp. & - & - & - & - & - & - & [110] \\
\hline \multirow[t]{5}{*}{2.} & Cadmium & Pseudomonas putida mt2 & - & - & - & - & - & - & [111-114] \\
\hline & & $\begin{array}{l}\text { Cupriavidus metallidurans } \\
\mathrm{CH} 34\end{array}$ & - & - & - & - & - & - & [111-114] \\
\hline & & Enterobacter cloacae & 25 & 5 & 240 & 2 & 0.1 & $58.9 \%$ & [115] \\
\hline & & Stenotrophomonas maltophilia & 28 & 5 & 140 & 2 & 20 & 0.12 & [116] \\
\hline & & Actinomycetes sp. & 30 & 6 & 150 & 24 & 5 & 32.63 & {$[116]$} \\
\hline \multirow[t]{6}{*}{3.} & Chromium & Micrococcus sp. & 35 & 5 & 120 & 24 & - & $92 \%$ & [117] \\
\hline & & Bacillus licheniformis & 28 & 3.5 & 120 & 48 & - & $95 \%$ & [116] \\
\hline & & Staphylococcus saprophyticus & 27 & 2 & 150 & 3 & 0.2 & 24.1 & {$[118]$} \\
\hline & & Enterobacter cloacae & 25 & 4 & 240 & 2 & 0.1 & 55.8 & [115] \\
\hline & & Pseudomonas aeruginosa & 25 & - & - & - & - & 1.07 & [119] \\
\hline & & Micrococcus sp. & 35 & 5 & 120 & 24 & - & $92 \%$ & {$[117]$} \\
\hline 4. & Cobalt & Rhodopseudomonas palustris & - & - & - & - & - & - & {$[120]$} \\
\hline \multirow[t]{8}{*}{5.} & Copper & Stenotrophomonas maltophilia & 25 & 5 & 140 & 2 & 20 & 0.57 & {$[116]$} \\
\hline & & Bacillus licheniformis & 28 & 2.5 & 120 & 48 & - & $32 \%$ & {$[121]$} \\
\hline & & $\begin{array}{l}\text { Geobacillus } \\
\text { thermodenitrificans }\end{array}$ & 25 & 5 & 100 & 12 & - & 51 & [122] \\
\hline & & Bacillus cereus & 25 & 5.5 & - & 24 & 1.0 & 50.32 & [119] \\
\hline & & Pseudomonas aeruginosa & 25 & - & - & - & - & 0.67 & [119] \\
\hline & & Thiobacillus thiooxidans & 30 & 5 & 786 & 2 & 0.25 & 39.84 & {$[123]$} \\
\hline & & Enterobacter cloacae & 25 & 5 & 240 & 2 & 0.1 & 78.9 & [115] \\
\hline & & Staphylococcus saprophyticus & 27 & 3.5 & 150 & 2 & 0.2 & 14.5 & [118] \\
\hline 6. & Gold & $\begin{array}{l}\text { Cupriavidus metallidurans } \\
\mathrm{CH} 34\end{array}$ & - & - & - & - & - & - & [125] \\
\hline
\end{tabular}




\begin{tabular}{|c|c|c|c|c|c|c|c|c|c|}
\hline $\begin{array}{l}\text { Sr. } \\
\text { No. }\end{array}$ & Metals & Bacteria & $\begin{array}{l}\text { Temperature } \\
\left({ }^{\circ} \mathrm{C}\right)\end{array}$ & $\mathrm{pH}$ & Agitation & Time & $\begin{array}{l}\text { Wt } \\
(\mathrm{g} / \mathrm{L})\end{array}$ & $\begin{array}{l}\mathrm{q}(\mathrm{mg} / \mathrm{g}) \text { or } \\
\% \text { removal }\end{array}$ & References \\
\hline \multirow[t]{7}{*}{7.} & Lead & Enterobacter cloacae & 25 & 5 & 240 & 2 & 0.1 & 67.9 & [115] \\
\hline & & Bacillus sp. & 30 & $5-9$ & 100 & 24 & - & 69.34 & [124] \\
\hline & & Pseudomonas sp. & 30 & $5-9$ & 100 & 24 & - & 90.41 & [124] \\
\hline & & Micrococcus sp. & 30 & $5-9$ & 100 & 24 & - & $84.27 \%$ & [124] \\
\hline & & Bacillus cereus & 25 & 5.5 & - & 24 & 1.0 & 36.71 & [119] \\
\hline & & $\begin{array}{l}\text { Geobacillus } \\
\text { thermodenitrificans }\end{array}$ & 25 & 4 & 100 & 12 & - & 53 & [122] \\
\hline & & Stenotrophomonas maltophilia & 25 & 5.0 & 140 & 2 & 20 & 0.41 & [116] \\
\hline 8. & Mercury & Enterobacter cloacae & 25 & 4 & 240 & 2 & 0.1 & 43.23 & [115] \\
\hline \multirow[t]{2}{*}{9.} & Nickel & Actinomycetes sp. & 30 & 5 & 150 & 24 & 5 & 36.55 & [116] \\
\hline & & Micrococcus sp. & 35 & 5 & 120 & 24 & - & $90 \%$ & [117] \\
\hline 10. & Selenium & $\begin{array}{l}\text { Cupriavidus metallidurans } \\
\text { CH34 }\end{array}$ & - & - & - & - & - & - & [111-114] \\
\hline 11. & Silver & $\begin{array}{l}\text { Cupriavidus metallidurans } \\
\text { CH34 }\end{array}$ & - & - & - & - & - & - & [111-114] \\
\hline \multirow[t]{2}{*}{12.} & Zinc & Pseudomonas aeruginosa & 25 & - & - & - & - & 1.33 & [119] \\
\hline & & $\begin{array}{l}\text { Geobacillus } \\
\text { thermodenitrificans }\end{array}$ & 25 & 5 & 100 & 12 & - & 18 & [122] \\
\hline
\end{tabular}

Where, Wt = weight of used adsorbent; $\mathrm{Q}=$ uptake removal of pollutant $(\mathrm{mg} / \mathrm{g})$; Agitation = speed of shaker $(\mathrm{rpm})$; $\mathrm{T}=$ Temperature of the experiment $\left({ }^{\circ} \mathrm{C}\right)$.

Table 2. Bacteria and their biosorption features regarding different metals [48, 126, 127].

\subsubsection{Algae}

Algae are aquatic plants that lack true roots and stems. It can range from micro algae to macroalgae. They are autotrophic. They can grow in big biomass even when less nutrition is provided. They are considered good biosorbent material [48, 70-73] because of their big size, high sorption capacity and no production of toxic substances. Mostly they are classified as microalgae (fresh water or green algae), macroalgae (marine or brown algae) and red algae. Among these three classes, brown alga is reported to have higher metal uptake capacity. The following features are responsible for binding of heavy metal ions to algae surface; algae species, ionic charge of metal and chemical composition of metal ion solution. Metal ion binding sites on algal surface includes sulfhydryl, hydroxyl, phosphate, sulfate, imidazole, amine, carboxyl groups [74]. The metal uptake mechanism of algae is similar to that of bacteria that is bonding of metal ions with the surface followed by internalization. According to Abbas et al., [48], either of two mechanisms in algal biosorption is involved: (1) ion exchange method where ions present on algal surface $\mathrm{Ca}, \mathrm{Mg}, \mathrm{Na}$, K they are displaced by metal ions, (2) complexation between functional groups and metal ions. 


\subsubsection{Biosorption by algae}

According to Abbas et al., [48], algal cell wall is made up of polysaccharides (alginic acid, chitin, xylan, mannan) which provides functional groups (sulfate, hydroxyl, phosphate, imidazole, amino, amine) known to act as metal binding sites [74]. As far as metal binding mechanism is concerned, ionic charge and covalent bonding are hypothesized. Carboxyl and sulfate groups are involved in ionic bonding whereas amino and carboxyl groups are involved in covalent bonding between metal ion and functional group. In response to metal ions, phytochelatins are produced inside the algal body [48]. Biosorption of various metals by different bacteria is given in Table 3 .

\begin{tabular}{|c|c|c|c|c|c|c|c|c|c|}
\hline $\begin{array}{l}\text { Sr. } \\
\text { No. }\end{array}$ & Metals & Algae & $\begin{array}{l}\text { Temperature } \\
\left({ }^{\circ} \mathrm{C}\right)\end{array}$ & $\mathrm{pH}$ & Agitation & Time & Wt $(g / L)$ & $\begin{array}{l}\mathrm{q}(\mathrm{mg} / \mathrm{g}) \text { or } \% \\
\text { removal }\end{array}$ & References \\
\hline 1. & Arsenic & Spirogyra hyalina & 25 & - & 180 & 2 & 1 & 9.8 & [128] \\
\hline 2. & Cadmium & $\begin{array}{l}\text { Bifurcaria } \\
\text { bifurcate } \\
\text { Oocystis } \\
\text { Pithophora spp. } \\
\text { (filamentous) } \\
\text { Sargassum sp. } \\
\text { (brown algae) } \\
\text { Sargassum } \\
\text { tenerrimum } \\
\text { Fucus vesiculosus } \\
\text { (brown algae) } \\
\text { Ascophyllum } \\
\text { nodosum }\end{array}$ & $\begin{array}{l}- \\
28 \\
25 \\
30 \\
25 \\
25 \\
25\end{array}$ & $\begin{array}{l}4.5 \\
7.5 \\
- \\
5 \\
6 \\
6 \\
6\end{array}$ & $\begin{array}{l}175 \\
72 \\
- \\
150 \\
150 \\
- \\
-\end{array}$ & $\begin{array}{l}3 \\
60-80 \\
9 \text { days } \\
- \\
24 \\
2 \\
2\end{array}$ & $\begin{array}{l}2.5 \\
28-51 \\
0.17-14 \\
- \\
4 \\
0.25 \\
0.5-1\end{array}$ & $\begin{array}{l}95 \\
- \\
- \\
22.2 \\
0.4 \mathrm{mmol} / \mathrm{g} \\
1.12 \mathrm{mmol} / \mathrm{g} \\
114.9\end{array}$ & $\begin{array}{l}{[129]} \\
{[130]} \\
{[131]} \\
{[132]} \\
{[133]} \\
{[134]} \\
{[135]}\end{array}$ \\
\hline 3. & Chromium & $\begin{array}{l}\text { Pithophora spp. } \\
\text { (filamentous) } \\
\text { Sargassum sp. } \\
\text { Spirogyra sp. } \\
\text { (green algae) } \\
\text { Sargassum sp. } \\
\text { (brown algae) }\end{array}$ & $\begin{array}{l}25 \\
30 \\
30 \\
30\end{array}$ & $\begin{array}{l}- \\
4 \\
4 \\
3\end{array}$ & $\begin{array}{l}- \\
- \\
180 \\
150\end{array}$ & $\begin{array}{l}9 \text { days } \\
6 \\
3 \\
-\end{array}$ & $\begin{array}{l}- \\
2-5 \\
1-3 \\
-\end{array}$ & $\begin{array}{l}- \\
68.9 \\
265 \\
20.2\end{array}$ & $\begin{array}{l}{[131]} \\
{[136]} \\
{[132]} \\
{[132]}\end{array}$ \\
\hline 4. & Cobalt & Spirogyra hyalina & 25 & - & 180 & 2 & 2.5 & 7.856 & [128] \\
\hline 5. & Copper & $\begin{array}{l}\text { Calotropis procera } \\
\text { Oocystis } \\
\text { Sargassum } \\
\text { filipendula } \\
\text { Microalgae } \\
\text { Sargassum sp. } \\
\text { (brown algae) } \\
\text { Fucus vesiculosus } \\
\text { (brown algae) } \\
\text { Ascophyllum } \\
\text { nodosum }\end{array}$ & $\begin{array}{l}25 \\
28 \\
25 \\
30 \\
30 \\
25 \\
25\end{array}$ & $\begin{array}{l}4 \\
5.5 \\
4.5 \\
- \\
4 \\
5 \\
4\end{array}$ & $\begin{array}{l}150 \\
60-80 \\
175 \\
150 \\
150 \\
- \\
-\end{array}$ & $\begin{array}{l}6 \\
72 \\
6 \\
- \\
- \\
2 \\
2\end{array}$ & $\begin{array}{l}2 \\
4.4-6.0 \\
5 \\
5 \\
- \\
0.25 \\
0.5-1\end{array}$ & $\begin{array}{l}14.5 \\
- \\
- \\
0.66 \\
18.6 \\
0.97 \\
70.9\end{array}$ & $\begin{array}{l}{[137]} \\
{[130]} \\
{[138]} \\
{[139]} \\
{[132]} \\
{[134]} \\
{[135]}\end{array}$ \\
\hline 7. & Lead & $\begin{array}{l}\text { Calotropis procera } \\
\text { Oocystis } \\
\text { Pithophora spp. } \\
\text { (filamentous) } \\
\text { Fucus vesiculosus } \\
\text { (brown algae) }\end{array}$ & $\begin{array}{l}25 \\
28 \\
25 \\
25\end{array}$ & $\begin{array}{l}4 \\
5.5 \\
- \\
5\end{array}$ & $\begin{array}{l}150 \\
60-80 \\
- \\
-\end{array}$ & $\begin{array}{l}6 \\
72 \\
9 \text { days } \\
2\end{array}$ & $\begin{array}{l}2 \\
16-80 \\
0.12-0.13 \\
0.25\end{array}$ & $\begin{array}{l}22.8 \\
- \\
- \\
1.04\end{array}$ & $\begin{array}{l}{[137]} \\
{[130]} \\
{[131]} \\
{[134]}\end{array}$ \\
\hline
\end{tabular}




\begin{tabular}{|c|c|c|c|c|c|c|c|c|c|}
\hline $\begin{array}{l}\text { Sr. } \\
\text { No. }\end{array}$ & Metals & Algae & $\begin{array}{l}\text { Temperature } \\
\left({ }^{\circ} \mathrm{C}\right)\end{array}$ & $\mathrm{pH}$ & Agitation & Time & Wt $(\mathrm{g} / \mathrm{L})$ & $\begin{array}{l}\mathrm{q}(\mathrm{mg} / \mathrm{g}) \text { or } \% \\
\text { removal }\end{array}$ & References \\
\hline 8. & Mercury & $\begin{array}{l}\text { Sargassum sp. } \\
\text { (brown algae) } \\
\text { Cladophora } \\
\text { fascicularis } \\
\text { Spirogyra hyaline }\end{array}$ & $\begin{array}{l}30 \\
25 \\
25\end{array}$ & $\begin{array}{l}4 \\
- \\
-\end{array}$ & $\begin{array}{l}100 \\
180 \\
180\end{array}$ & $\begin{array}{l}- \\
2 \\
2\end{array}$ & $\begin{array}{l}- \\
1 \\
2.5\end{array}$ & $\begin{array}{l}14.8 \\
20 \\
39.2\end{array}$ & $\begin{array}{l}{[132]} \\
{[128]} \\
{[128]}\end{array}$ \\
\hline 9. & Nickel & $\begin{array}{l}\text { Sargassum sp. } \\
\text { (brown algae) } \\
\text { Fucus vesiculosus } \\
\text { (brown algae) } \\
\text { Ascophyllum } \\
\text { nodosum }\end{array}$ & $\begin{array}{l}30 \\
25 \\
25\end{array}$ & $\begin{array}{l}5 \\
5 \\
6\end{array}$ & $\begin{array}{l}150 \\
- \\
-\end{array}$ & $\begin{array}{l}- \\
2 \\
2\end{array}$ & $\begin{array}{l}- \\
0.25 \\
0.5-1\end{array}$ & $\begin{array}{l}26.1 \\
0.80 \\
50\end{array}$ & $\begin{array}{l}{[132]} \\
{[134]} \\
{[135]}\end{array}$ \\
\hline 12. & Zinc & $\begin{array}{l}\text { Microalgae } \\
\text { Sargassum sp. } \\
\text { (brown algae) } \\
\text { Ascophyllum } \\
\text { nodosum }\end{array}$ & $\begin{array}{l}30 \\
30 \\
25\end{array}$ & $\begin{array}{l}- \\
3 \\
6\end{array}$ & $\begin{array}{l}150 \\
150 \\
-\end{array}$ & $\begin{array}{l}- \\
- \\
2\end{array}$ & $\begin{array}{l}5 \\
- \\
0.5-1\end{array}$ & $\begin{array}{l}0.72 \mathrm{mmol} / \mathrm{g} \\
15.4 \\
53.2\end{array}$ & $\begin{array}{l}{[139]} \\
{[132]} \\
{[135]}\end{array}$ \\
\hline 13. & Iron & $\begin{array}{l}\text { Sargassum sp. } \\
\text { (brown algae) }\end{array}$ & 30 & 3 & 150 & - & - & 14.6 & [132] \\
\hline
\end{tabular}

Table 3. Algae and their biosorption features regarding different metals [48].

\subsubsection{Fungi}

Fungi are eukaryotic living organism which includes yeasts, mushrooms, molds, etc. The cell wall structure of fungi offers good metal binding properties. Fungi in living and dead both forms can be used as biosorbent material $[48,75]$. Metal uptake by fungi involves two processes (i) active uptake or bioaccumulation or intracellular uptake, it is dependent on cell metabolism and (ii) biosorption or passive uptake which involves binding of metal ions to surface of cell wall and it is independent of cell metabolism. The energy independent metal uptake mechanism can be affected by temperature, metabolic inhibitors, etc. Metal uptake by fungi was reported both active and passive. Active uptake occurred only with living cells. In this case, the interaction of metal ions with cell surface functional groups may involves ionexchange, complexation or just physical adsorption.

\subsubsection{Biosorption by fungi}

According to Das et al., [69] fungal cell wall exhibit excellent metal biding properties due to its components. The cell wall of fungus is composed mainly of chitins, mannans, glucans, in addition to lipids, polysaccharides, pigments e.g. melanin [48, 76-78]. Fungal cell wall is reported to be made up of $90 \%$ polysaccharides. The functional groups which are involved in metal binding includes carboxyl, phosphate, uranic acids, proteins, nitrogen containing ligands, chitin or chitosan [48, 79]. Biosorption ability of fungal cells can be manipulated by physical of chemical treatments including autoclaving, heat processes or dimethyl sulfoxide, laundry detergent, orthophosphoric acid, formaldehyde, gluteraldehyde, $\mathrm{NaOH}$, respectively [69]. Macrofungi also called as mushrooms, grow wild in all types of environments 
ranging from forests to polluted soils and water bodies. They uptake the metals in their fruiting bodies, mycelia and sporocarps [48]. Biosorption of various metals by different fungi and mushrooms is given in Tables 4 and 5 respectively.

\subsubsection{Yeasts}

Yeasts are famous organisms while studying biosorption. Saccharomyces cerevisiae is well known yeast which is considered a model system to study biosorption. They are easy to grow, non-pathogenic and give high biomass yield using simple growth medium [80]. The availability of complete genome information makes its genetic engineering an easy job $[75,81]$. They are also considered ideal experimental organism in molecular biology experimentation $[75,82-84]$. The property of biosorption by yeast cells is affected by various factors including properties of metal ions (valency, radius), cell age of $S$. cerevisiae cells, conditions of culture (composition of growth medium, carbon source), biosorption conditions (initial concentration of metals and biomass, availability of metal ions, temperature, $\mathrm{pH}$, other ions in growth medium) [75]. Moreover, the large size of yeast makes them promising candidates for metal bioremediation. Saccharomyces cerevisiae is a widely studied yeast strain. Its different forms are already studied for its biosorption properties including immobilized versus fess cell, living versus dead cells, engineered versus non engineered cells, cultural versus waste cells, etc. [69, 85-89].

\begin{tabular}{|c|c|c|c|c|c|c|c|c|c|}
\hline $\begin{array}{l}\text { Sr. } \\
\text { No. }\end{array}$ & Metals & Bacteria & $\begin{array}{l}\text { Temperature } \\
\left({ }^{\circ} \mathrm{C}\right)\end{array}$ & $\mathrm{pH}$ & Agitation & Time & $\begin{array}{l}\text { Wt } \\
(\mathrm{g} / \mathrm{L})\end{array}$ & $\begin{array}{l}\mathrm{q}(\mathrm{mg} / \mathrm{g}) \text { or } \\
\% \text { removal }\end{array}$ & References \\
\hline 1. & Arsenic & Penicillium chrysogenum & 25 & $3-4$ & 190 & - & 1 & 24.5 & {$[140]$} \\
\hline \multirow[t]{3}{*}{2.} & \multirow[t]{3}{*}{ Cadmium } & Aspergillus cristatus & 25 & 6 & 120 & 2 & 0.4 & 23.2 & [142] \\
\hline & & Aspergillus niger & 25 & 4.75 & 125 & 6 & 0.7 & 13 & [143] \\
\hline & & Hydrilla verticillata & 25 & 5 & 150 & 0.33 & $3-9$ & 15 & {$[141]$} \\
\hline \multirow[t]{5}{*}{3.} & \multirow[t]{5}{*}{ Chromium } & Aspergillus niger & 28 & 4.5 & 150 & 1 & 10 & 16.39 & {$[144,145]$} \\
\hline & & Pleurotus ostreatus & 25 & 4.5 & 150 & 3 & 2 & 1.97 & [146] \\
\hline & & Trichoderma viride & - & 6 & 150 & 0.75 & 3.75 & 4.66 & [147] \\
\hline & & Mucor & 35 & 5.5 & - & - & - & - & [148] \\
\hline & & Penicillium canescens & 20 & 6 & 100 & 4 & 2 & 34.8 & [149] \\
\hline \multirow[t]{3}{*}{4.} & \multirow[t]{3}{*}{ Copper } & Pleurotus ostreatus & 25 & 4.5 & 150 & 3 & 2 & 4.0 & {$[150]$} \\
\hline & & Fomes fasciatus & 25 & 5.5 & 200 & 1 & 1 & 32.2 & {$[151]$} \\
\hline & & Aspergillus lentulus & 35 & 6 & 180 & 0.41 & 4 & - & {$[152]$} \\
\hline \multirow[t]{3}{*}{5.} & \multirow[t]{3}{*}{ Lead } & Rhizopus nigricans & 25 & 5.5 & 225 & - & 25 & 80.8 & {$[153]$} \\
\hline & & Trichoderma & 25 & 7 & - & 0.33 & - & 71 & [154] \\
\hline & & $\begin{array}{l}\text { longibrachiatum } \\
\text { Pleurotus ostreatus }\end{array}$ & 25 & 5.5 & - & 3 & 2 & 4.84 & [155] \\
\hline \multirow[t]{2}{*}{6.} & \multirow[t]{2}{*}{ Mercury } & Aspergillus flavus & 30 & 5.5 & 100 & 8 & 10 & $95.3 \%$ & [156] \\
\hline & & Aspergillus fumigatus & 30 & 5.5 & 100 & 8 & 10 & $95.3 \%$ & [140] \\
\hline 7. & Nickel & Aspergillus niger & 25 & 4.5 & 150 & 3 & 1 & 7.69 & [157] \\
\hline
\end{tabular}

Table 4. Fungi and their biosorption features regarding different metals [48]. 


\begin{tabular}{llll}
\hline Sr. No. & Mushrooms & Metals & References \\
\hline 1. & Volvariella volvacea (edible Mushroom) - mycelia, & Cadmium, lead, Copper, & {$[158]$} \\
sporocarps & Chromium & {$[69,159]$} \\
2. & Ganoderma lucidum & Chromium & {$[160]$} \\
3. & Coriolopsis strumosa & Copper & {$[160]$} \\
4. & Daedalea tenuis & Copper & {$[160]$} \\
5. & Lentinus strigosus & Copper & {$[160]$} \\
6. & Lenzites malaccensis & Copper & {$[160]$} \\
7. & Phellinus xeranticus & Copper & {$[160]$} \\
8. & Rigidoporus lineatus & Copper & {$[160]$} \\
9. & Rigidoporus microporus & Copper & {$[160]$} \\
10. & Trametes lactinea & Copper & {$[159,160]$} \\
11. & Ganoderma lucidum & Copper & {$[161]$} \\
12. & Agaricus macrospores & Cadmium, mercury, copper & . \\
\hline
\end{tabular}

Table 5. Mushrooms and biosorption of different metals [48].

\subsubsection{Biosorption by yeast}

The free form of yeast cells is not considered good candidates for biosorption [86]. Free cells face the problem of separation of solid liquid phase. This problem seems to be less effective in flocculating cell [90]. Pretreatment of yeast cells can result in increased surface to volume ration for binding of metal with the metal binding sites. It is reported that $\mathrm{pH}$ above 5 optimizes the metal biosorption in yeast cells [91]. According to Abbas et al., [48] in yeasts, higher concentration of heavy metals can be accumulated by bioaccumulation process than biosorption. However, general biosorption is responsible for the major uptake of heavy metals for many filamentous fungi. Biosorption of various metals by different yeasts is given in Table 6 .

\begin{tabular}{|c|c|c|c|c|c|c|c|c|c|}
\hline $\begin{array}{l}\text { Sr. } \\
\text { No. }\end{array}$ & Metals & Yeasts & $\begin{array}{l}\text { Temperature } \\
\left({ }^{\circ} \mathrm{C}\right)\end{array}$ & $\mathrm{pH}$ & Agitation & Time & $\begin{array}{l}W t \\
(g / L)\end{array}$ & $\begin{array}{l}\mathrm{q}(\mathrm{mg} / \mathrm{g}) \text { or } \\
\% \text { removal }\end{array}$ & References \\
\hline 1. & Cadmium & Saccharomyces cerevisiae & 25 & 7 & 100 & 2 & 2 & 12.3 & [69] \\
\hline 2. & Chromium & $\begin{array}{l}\text { Saccharomyces cerevisiae } \\
\text { Candida utilis }\end{array}$ & $\begin{array}{l}25 \\
25\end{array}$ & $\begin{array}{l}5.2 \\
5.5\end{array}$ & $\begin{array}{l}150 \\
160\end{array}$ & $\begin{array}{l}1 \\
1\end{array}$ & $\begin{array}{l}80 \\
1.0\end{array}$ & $\begin{array}{l}55.3 \% \\
28\end{array}$ & $\begin{array}{l}{[162]} \\
{[162]}\end{array}$ \\
\hline 3. & Cobalt & Saccharomyces cerevisiae & 25 & 7 & 100 & 2 & 2 & 8.2 & {$[162,163]$} \\
\hline 4. & Copper & $\begin{array}{l}\text { Saccharomyces cerevisiae } \\
\text { Candida pelliculosa } \\
\text { Schizosaccharomyces pombe }\end{array}$ & $\begin{array}{l}25 \\
30 \\
25\end{array}$ & $\begin{array}{l}7 \\
6 \\
4\end{array}$ & $\begin{array}{l}100 \\
120 \\
-\end{array}$ & $\begin{array}{l}2 \\
120 \\
96\end{array}$ & $\begin{array}{l}2 \\
13.3 \\
-\end{array}$ & $\begin{array}{l}29.9 \\
95.04 \% \\
74.85\end{array}$ & $\begin{array}{l}{[162]} \\
{[164]} \\
{[165]}\end{array}$ \\
\hline 5. & Lead & Mucor rouxii & 25 & 5.0 & 125 & 15 & - & 17.13 & {$[166]$} \\
\hline 6. & Mercury & Saccharomyces cerevisiae & 25 & 7 & 100 & 2 & 2 & 76.2 & {$[162]$} \\
\hline 7. & Nickel & Saccharomyces cerevisiae & 25 & 7 & 100 & 2 & 2 & 14.1 & {$[162]$} \\
\hline 8. & Zinc & Saccharomyces cerevisiae & 25 & 7 & 100 & 2 & 2 & 11.8 & [162] \\
\hline
\end{tabular}

Table 6. Yeasts and their biosorption features regarding different metals [48]. 


\subsection{Non-living organic materials}

\subsubsection{Wastes of agricultural or food industry}

The wastes of agriculture or food industry includes agricultural byproducts as corn cobs, soya bean hulls, cotton seeds hulls [92] or fruit peels. They contain cellulosic material in their cell wall which is known to contain functional groups like phenolics or carboxylic. On the basis of cation exchange between functional groups and metal ions, the binding of metal ion with functional group results in biosorption and thus removal of metal ion from medium [49].

\section{Factors affecting biosorption}

Biosorption process is affected by following factors.

Temperature: For efficient removal of metal ions from environment, the optimum temperature needed to be investigated. It is generally assumed that biosorption is carried out between 20 and $35^{\circ} \mathrm{C}$. High temperatures above $45^{\circ} \mathrm{C}$ may results in damage to proteins which in turn affects metal uptake process [48, 93-95].

pH: It is a very important parameter. It affects solubility of metal ions and binding sites of biomass. At lower $\mathrm{pH}$, the biosorption of metals is affected [96, 97]. General range of $\mathrm{pH}$ for metal uptake is between 2.5-6. Above this limit, metal uptake ability of biosorbent gets compromised [48].

Nature of biosorbents: Metal uptake is reported in different forms like biofilms, freely suspended microbial cells or immobilization of microbial cells. It can be altered by physical or chemical treatments. Physical treatments include autoclaving, drying, boiling, sonication, etc. Chemical treatment as the name indicates involves chemicals like acid or alkali to improve biosorption capacity. According to Wang and Chen, [75], the fungal cells are deacetylated which affects the structure of chitin resulting in the formation of chitosan-glycan complexes which have results high metal affinities. Abbas et al., [48] also report about effect of age, growth medium components on biosorption as they might result in cell wall composition, cell size and EPS formation.

Surface area to volume ratio: This property plays an important role in efficient removal of heavy metal from medium. The surface area property plays a significant role in case of biofilms [48]. The binding of metal ions with microbial cell wall is previously reported [98]. Although intracellular metal adsorption is energy-consuming process but still microorganisms prefer it over wall adsorption.

Concentration of biomass: The concentration of biomass is directly proportional to the metal uptake $[48,98,99]$. It is reported that electrostatic interaction between the cells plays an important role in metal uptake. At a given equilibrium, the biomass adsorbs more metal ions at low cell densities than at high densities [100]. Metal uptake depends on biding sites. More biomass concentration or more metal ions restricts the access of metal ions to binding sites $[48,101]$. 
Initial metal ion concentration: The initial concentration provides an important driving force to overcome all mass transfer resistance of metal between the aqueous and solid phases [102]. Increasing amount of metal adsorbed by the biomass will be increased with initial concentration of metals. Optimum percentage of metal removal can be taken at low initial metal concentration. Thus, at a given concentration of biomass, the metal uptake increases with increase in initial concentration [48].

Metal affinity to biosorbent: Physical/chemical pretreatment affects permeability and surface charges of the biomass and makes metal binding groups accessible for binding. It can be manipulated by pretreating the biomass with alkalis, acids detergents and heat, which may increase the amount of metal uptake [48, 94].

\section{Kinetics of biosorption}

Before going in the details of studying kinetics of biosorption, one should understand the quality of a biosorbent. For observing the quality of a biosorbent, two factors should be considered (i) how much metal ion is attracted by the biosorbent, (ii) to which extent metal ions are retained on biosorbent in an immobilized form. The metal uptake by the biosorbent can be calculated by checking the difference in initial quantities of metal ions in medium to that remained in the medium after biosorption takes place. This is studied by the following Eq. 1 [48, 49, 94]:

$$
q=\frac{V(C i-C e)}{M}
$$

$\mathrm{q}=$ amount of metal biosorbed by biomass (mg/g); V = Volume of metal solution (L); Ci = Initial concentration of metal $(\mathrm{mg} / \mathrm{L}) ; \mathrm{Ce}=$ Concentration of metal $(\mathrm{mg} / \mathrm{L})$ at equilibrium; $\mathrm{M}=$ Mass of adsorbent.

Units = milligrams of solute sorbed per gram of dry biosorbent material (when engineering process - mass balance calculations are to be considered) or $\mathrm{mmol} / \mathrm{g}$ (when the mechanism or stoichiometry are to be considered).

According to Abdi and Kazemi [49], in order to observe biosorption kinetics of any heavy metal, sorption performance of a biosorbent must be taken into consideration. For it, a biosorption isotherm should be studied. A biosorption isotherm is the plot of uptake of metal (q) versus equilibrium solute concentration in the solution (Cf). For studying the isotherm plots, parameters including temperature, $\mathrm{pH}$ and ionic strength are kept constant whereas metal concentration is varied. Literature showed that confusion prevails regarding $\mathrm{pH}$ because it is common believe that $\mathrm{pH}$ of a medium changes during whole process of biosorption. Biosorption isotherms are typically described by two models (i) Freundlich and (ii) Langmuir. These models are two - parameters models which are vastly used to describe the equilibrium state for adsorption of metal ions experimental work [48].

Freundlich model: Freundlich and Kuster in (1907) published first mathematical equation to describe the isotherm. It is a non-liner sorption model. It involves monolayer sorption of 
metal with active sites and is described by continuous interactions between adsorbed molecules $[49,103]$. It is given by Eq. 2 :

$$
q e=K C e_{n}^{1}
$$

$\mathrm{K}=\mathrm{mg} / \mathrm{g}$ or $\mathrm{l} / \mathrm{mg} ; 1 / \mathrm{n}$ or $\mathrm{n}=$ Freundlich constant related to adsorption capacity; $\mathrm{n}=$ Freundlich constant related to adsorption intensity.

Langmuir model: Langmuir in 1918 published a model for describing gas or liquid adsorbed on solid material. It describes the monolayer sorption of metal with active sites and do not involve interactions between adsorbed molecules [48, 49]. It is given by Eq. 3:

$$
q e=\frac{q \max b C e}{1+b C e}
$$

$\mathrm{qe}=$ Amount of metal ion removed $(\mathrm{mg} / \mathrm{g}) ; \mathrm{Ce}=$ Equilibrium concentration $(\mathrm{mg} / \mathrm{L}) ;$ $\mathrm{b}=$ Langmuir constant related to affinity; qmax = maximum metal uptake $(\mathrm{mg} / \mathrm{g})$ under the given conditions.

$\mathrm{k}, \mathrm{n}=$ Freundlich and Langmuir constants ( $\mathrm{n}$ value greater than 1.0 shows that sorption is favorable physical process) $[49,104]$.

\section{Desorption and recovery of metals}

After biosorption of heavy metal from environment, its recovery is another crucial step which involves desorption of metal from biosorbent. According to previous literatures [105-107], various agents were used for this purpose which includes complexing agents (thiosulfate, EDTA), mineral acids $\left(\mathrm{HNO}_{3}, \mathrm{H}_{2} \mathrm{SO}_{4}, \mathrm{HCl}\right)$, organic acids (acetic acid, citric acid). Before choosing the recovery agents, it should be kept in mind that chosen recovery agent should given least harm to physical properties of a biosorbent so that its efficiency of metal binding must remain in its original state to ensure its maximum efficiency for metal binding [94, 106, 107].

\section{Conclusions}

Biosorption is eco-friendly and cheap method of removing metals from the environment. Previous researches conducted during last five decades provided vast amount of information about different types of biosorbents and their mechanism of metal uptake. More research is needed to explore new biosorbents from environment. A deep insight is required not only on method of metal removal, but also its efficient recovery so that it can be obtained in usable form. 


\section{Author details}

Saba Shamim

Address all correspondence to: sabashamimgenetics@gmail.com

Institute of Molecular Biology and Biotechnology (IMBB), The University of Lahore (UOL), Lahore, Pakistan

\section{References}

[1] Shamim S. Comparative analysis of metal resistance, accumulation and antioxidant enzymes in Cupriavidus metallidruans $\mathrm{CH} 34$ and Pseudomonas putida mt2 during cadmium stress. Ph. D. thesis. Department of Microbiology and Molecular Genetics, University of the Punjab: Pakistan; 2016

[2] Fulekar MH, Singh A, Bhaduri AM. Genetic engineering strategies for enhancing phytoremediation of heavy metals. African Journal of Biotechnology. 2009;8(4):529-535

[3] Shokrzadeh M, Saravi SSS. The study of heavy metals (zinc, lead, cadmium, and chromium) in water sampled from Gorgan coast (Iran). Toxicology and Environmental Chemistry. 2009;91(3):405-407. DOI: 10.1080/02772240902830755

[4] Tálos K, Páger C, Tonk S, Majdik C, Kocsis B, Kilár F, Pernyeszi T. Cadmium biosorption on native Saccharomyces cerevisiae cells in aqueous suspension. Agriculture and Environment. 2009;1:20-30

[5] Joo JH, Hassan SHA, Oh SE. Comparative study of biosorption of $\mathrm{Zn}^{2+}$ by Pseudomonas aeruginosa and Bacillus cereus. International Biodeterioration and Biodegradation. 2010;64:734-741. DOI: 10.1016/j.ibiod.2010.08.007

[6] Kermani AJN, Ghasemi MF, Khosravan A, Farahmand A, Shakibaie MR. Cadmium bioremediation by metal-resistant mutated bacteria isolated from active sludge of industrial effluent. Iranian Journal of Environmental Health Science and Engineering. 2010;7(4):279-286

[7] Singh JS, Abhilash PC, Singh HB, Singh RP, Singh DP. Genetically engineered bacteria: An emerging tool for environmental remediation and future research perspectives. Gene. 2011;480:1-9. DOI: 10.1016/j.gene.2011.03.001

[8] Janssen PJ, Houdt RV, Moors H, Monsieurs P, Morin N, Michaux A, Benotmane MA, Leys N, Vallaeys T, Lapidus A, Monchy S, Médigue C, Taghavi S, McCorkle S, Dunn J, van der Lelie D, Mergeay M. The complete genome sequence of Cupriavidus metallidurans strain $\mathrm{CH} 34$, a master survivalist in harsh and anthropogenic environments. PLoS One. 2010;5(5):1-33. DOI: 10.1371/journal.pone.0010433 
[9] Kang SH, Singh S, Kim JY, Lee W, Mulchandani A, Chen W. Bacteria metabolically engineered for enhanced phytochelatin production and cadmium accumulation. Applied and Environmental Microbiology. 2007;73(19):6317-6320. DOI: 10.1128/AEM. 01237-07

[10] El-Sherif IY, Ashmawy A, Badr S. Biosorption of cadmium and nickel by Nile water algae. Journal of Applied Sciences Research. 2008;4(4):391-396

[11] Zhang H, Dang Z, Zheng LC, Yi XY. Remediation of soil co-contaminated with pyrene and cadmium by growing maize (Zea mays L.). International Journal of Environmental Science and Technology. 2009;6(2):249-258

[12] Ike A, Sriprang R, Ono H, Murooka Y, Yamashita M. Bioremediation of cadmium contaminated soil using symbiosis between leguminous plant and recombinant rhizobia with the MTL4 and the PCS genes. Chemosphere. 2007;66:1670-1676. DOI: 10.1016/j. chemosphere.2006.07.058

[13] Siñeriz ML, Kothe E, Abatel CM. Cadmium biosorption by Streptomyces sp. F4 isolated from former uranium mine. Journal of Basic Microbiology. 2009;49:55-62. DOI: 10.1002/ jobm. 200700376

[14] Pagnanelli F, Viggi CC, Toro L. Isolation and quantification of cadmium removal mechanisms in batch reactors inoculated by sulphate reducing bacteria: Biosorption versus bioprecipitation. Bioresource Technology. 2010;101:2981-2987. DOI: 10.1016/j. biortech.2009.12.009

[15] Nies DH. Microbial heavy-metal resistance. Applied Microbiology and Biotechnology. 1999;51:730-750

[16] Grosse C, Anton A, Hoffmann T, Franke S, Schleuder G, Nies DH. Identification of a regulatory pathway that controls the heavy-metal resistance system Czc via promoter czcNp in Ralstonia metallidurans. Archives of Microbiology. 2004;182(2-3):109-118. DOI: 10.1007/s00203-004-0670-8

[17] Nies DH. The elements: Essential and toxic effects on microorganisms. In: Anke K, Ihnat M, Stoeppler M, editors. Metals and their Compounds in the Environment, Part II. Weinheim: John Wiley; 2004

[18] Rehman A. Survey of protozoan fauna surviving in stress conditions in industrial wastes and their role in bioremediation - Ph. D. thesis. Department of Zoology, University of the Punjab, Lahore; 2006

[19] Weast RC. CRC Handbook of Chemistry and Physics. 64th ed. Boca Raton: CRC; 1984

[20] Zhang Y, Zhang H, Li X, Su Z, Zhang C. The cadA gene in cadmium-resistant bacteria from cadmium-polluted soil in the Zhangshi area of northeast China. Current Microbiology. 2008;56(3):236-239. DOI: 10.1007/s00284-007-9064-x 
[21] Kalantari N. Evaluation of toxicity of iron, chromium and cadmium on Bacillus cereus growth. Iranian Journal of Basic Medical Sciences. 2008;10(4):222-228

[22] Norton L, Baskaran K, McKenzie T. Biosorption of zinc from aqueous solutions using biosolids. Advances in Environmental Research. 2004;8:629-635

[23] Crini G. Non-conventional low-cost adsorbents for dye removal: A review. Bioresource Technology. 2006;97:1061-1085. DOI: 10.1016/j.biortech.2005.05.001

[24] Costa MC, Martins M, Jesus C, Duarte JC. Treatment of acid mine drainage by sulphatereducing bacteria using low cost matrices. Water, Air, and Soil Pollution. 2008;189: $149-162$

[25] Chen G, Zeng G, Tang L, Du C, Jiang X, Huang G, Liu H, Shen G. Cadmium removal from simulated wastewater to biomass byproduct of Lentinus edodes. Bioresource Technology. 2008;99(15):7034-7040. DOI: 10.1016/j.biortech.2008.01.020

[26] Tang L, Zeng GM, Shen GL, Li YP, Zhang Y, Huang DL. Rapid detection of picloram in agricultural field samples using a disposable immunomembrane based electrochemical sensor. Environmental Science and Technology. 2008;42:1207-1212

[27] Malik A. Metal bioremediation through growing cells. Environment International. 2004;30:261-278. DOI: 10.1016/j.envint.2003.08.001

[28] Tabak H, Lens P, Hullebusch EV, Dejonghe W. Developments in bioremediation of soils and sediments polluted with metals and radionuclides-1. Microbial processes and mechanisms affecting bioremediation of metal contamination and influencing metal toxicity and transport. Reviews in Environmental Science and Bio/Technology. 2005; 4:115-156

[29] Gadd GM. Biosorption: Critical review of scientific rationale, environmental importance and significance for pollution treatment. Journal of Chemical Technology and Biotechnology. 2009;84:13-28

[30] Choudhary S, Sar P. Characterization of a metal resistant Pseudomonas sp. isolated from uranium mine for its potential in heavy metal $\left(\mathrm{Ni}^{2+}, \mathrm{Co}^{2+}, \mathrm{Cu}^{2+}\right.$, and $\left.\mathrm{Cd}^{2+}\right)$ sequestration. Bioresource Technology. 2009;100:2482-2492. DOI: 10.1016/j.biortech.2008.12.015

[31] Hajdu R, Pinheiro JPR, Galceran J, Slaveykova VI. Modeling of Cd uptake and efflux kinetics in metal-resistant bacterium Cupriavidus metallidurans. Environmental Science and Technology. 2010;44:4597-4602. DOI: 10.1021/es100687h

[32] Volesky B. Biosorption process simulation tools. Hydrometallurgy. 2003;71:179-190

[33] Gabr RM, Hassan SHA, Shoreit AAM. Biosorption of lead and nickel by living and non-living cells of Pseudomonas aeruginosa ASU 6a. International Biodeterioration and Biodegradation. 2008;62:195-203 
[34] Hassan SHA, Kim SJ, Jung AY, Joo JH, SE O, Yang JE. Biosorptive capacity of Cd(II) and $\mathrm{Cu}(\mathrm{II})$ by lyophilized cells of Pseudomonas stutzeri. Journal of General and Applied Microbiology. 2009;55:27-34

[35] Vasudevan P, Padmavathy V, Dhingra SC. Kinetics of biosorption of cadmium on Baker's yeast. Bioresource Technology. 2003;89:281-287

[36] Cruz C, Costa A, Hemriques CA, Luna AS. Kinetic modeling and equilibrium studies during cadmium adsorption by dead Sargassum sp. biomass. Bioresource Technology. 2004;91:249-257

[37] Dostalek P, Patzak M, Matejka P. Influence of specific growth limitation on biosorption of heavy metals by Saccharomyces cerevisiae. International Biodeterioration and Biodegradation. 2004;54:203-207

[38] Göksungur Y, Üren S, Güvenç U. Biosorption of cadmium and lead ions by ethanol treated waste baker's yeast biomass. Bioresource Technology. 2005;96:103-109. DOI: 10.1016/j.biortech.2003.04.002

[39] Ledrich ML, Stemmler S, Laval-Gilly P, Foucaud L, Falla J. Precipitation of silver-thiosulfate complex and immobilization of silver by Cupriavidus metallidurans $\mathrm{CH} 34$. Biometals. 2005;18:643-650. DOI: 10.1007/s10534-005-3858-8

[40] Wang J, Chen C. Biosorption of heavy metals by Saccharomyces cerevisiae: A review. Biotechnology Advances. 2006;24:427-451. DOI: 10.1016/j.biotechadv.2006.03.001

[41] Ghorbani F, Younesi H, Ghasempouri SM, Zinatizadeh AA, Amini M, Daneshi A. Appli cation of response surface methodology for optimization of cadmium biosorption in an aqueous solution by Saccharomyces cerevisiae. Chemical Engineering Journal. 2008; 145:267-275

[42] Fereidouni M, Daneshi A, Younesi H. Biosorption equilibria of binary Cd(II) and Ni(II) systems onto Saccharomyces cerevisisae and Ralstonia eutropha cells: Application of response surface methodology. Journal of Hazardous Materials. 2009;2(3):1437-1448. DOI: 10.1016/j. jhazmat.2009.03.041

[43] Volesky B, Schiewer S. Biosorption, metals. In: Flickinger MC, Drew SW, editors. Encyclopedia of Bioprocess Technology: Processes Fermentation Biocatyalysis and Biosorption. New York: John Wiley and Sons; 2000. pp. 433-453

[44] Valls M, de Lorenzo V. Exploiting the genetic and biochemical capacities of bacteria for the remediation of heavy metal pollution. FEMS Microbiology Reviews. 2002;26: 327-338

[45] Sinha S, Mukherjee SK. Pseudomonas aeruginosa KUCD1, a possible candidate for cadmium bioremediation. Brazilian Journal of Microbiology. 2009;40:655-662. DOI: 10.1590/ S1517-838220090003000030 
[46] Lima AIG, Corticeiro SC, Figueira EMAP. Glutathione-mediated cadmium sequestration in Rhizobium leguminosarum. Enzyme and Microbial Technology. 2006;39(4):763-769

[47] Aksu Z, Sag Y, Kutsal T. The biosorption of copper by C. vulgaris and Z. ramigera. Environmental Technology. 1992;13:579-586

[48] Abbas SH, Ismail IM, Mostafa TM, Sulaymon AH. Biosorption of heavy metals: A review. Journal of Chemical Science and Technology. 2014;3(4):74-102

[49] Abdi O, Kazemi MA. Review study of biosorption of heavy metals and comparison between different biosorbents. Journal of Materials and Environmental Science. 2015;6(5):1386-1399

[50] Chen XC, Wang YP, Lin Q, Shi JY, WX W, Chen YX. Biosorption of copper(II) and zinc(II) from aqueous solution by Pseudomonas putida CZ1. Colloids and Surfaces B: Biointerfaces. 2005;46(2):101-107. DOI: 10.1016/j.colsurfb.2005.10.003

[51] Akhtar N, Iqbal M, Zafar SI, Iqbal J. Biosorption characteristics of unicellular green alga Chlorella sorokiniana immobilized in loofa sponge for removal of $\mathrm{Cr}(\mathrm{III})$. Journal of Environmental Sciences. 2008;20:231-239

[52] Galceran J, van Leeuwen HP. Dynamics of biouptake processes: The role of transport, adsorption and internalisation. In: van Leeuwen HP, Köster W, editors. Physicochemical Kinetics and Transport at Biointerfaces. Chichester: John Wiley and Sons; 2004. pp. 147203. DOI: $10.1002 / 0470094044 . c h 4$

[53] Wilkinson KJ, Buffle J. Critical evaluation of physicochemical parameters and processes for modelling the biological uptake of trace metals in environmental (aquatic) systems. In: Van Leeuwen HP, Koester W, editors. Physicochemical Kinetics and Transport at Biointerfaces. Chichester: John Wiley and Sons; 2004. pp. 447-533

[54] Volesky B. Advances in biosorption of metals: Selection of biomass types. FEMS Microbiology Reviews. 1994;14:291-302

[55] Bruins MR, Kapil S, Oehme FW. Microbial resistance to metals in the environment. Ecotoxicology and Environmental Safety. 2000;45:198-207. DOI: 10.1006/eesa.1999.1860

[56] Nies DH. Efflux-mediated heavy metal resistance in prokaryotes. FEMS Microbiology Reviews. 2003;27:313-339

[57] Diels L, Spaans P, Van Roy S, Hooyberghs L, Ryngaert A, Wouters H, Walter E, Winters J, Macaskie L, Finlay J, Pernfuss B, Woebking H, Pumpel T, Tsezos M. Heavy metals removal by sand filters inoculated with metal sorbing and precipitating bacteria. Hydrometallurgy. 2003;71:235-241

[58] Shamim S, Rehman A. Cadmium resistance and accumulation potential of Klebsiella Pneumoniae strain CBL-1 isolated from industrial wastewater. Pakistan Journal of Zoology. 2012;44(1):203-208 
[59] Campbell PGC, Errecalde O, Fortin C, Hiriart-Baer VP, Vigneault B. Metal bioavailability to phytoplankton-applicability of the biotic ligand model. Comparative Biochemistry and Physiology Part C: Toxicology and Pharmacology. 2002;133:189-206

[60] Pabst MW, Miller CD, Dimkpa CO, Anderson AJ, McLean JE. Defining the surface adsorption and internalization of copper and cadmium in a soil bacterium, Pseudomonas putida. Chemosphere. 2010;81:904-910. DOI: 10.1016/j.chemosphere.2010.07.069

[61] Nies DH, Silver S. Ion efflux systems involved in bacterial metal resistances. Journal of Industrial Microbiology. 1995;14:186-199

[62] Norberg A, Persson H. Accumulation of heavy metal ions by Zoogloea ramigera. Biotechnology and Bioengineering. 1984;26:239-245. DOI: 10.1002/bit.260260307

[63] Wang JL, Chen C. Biosorbents for heavy metals removal and their future - a review. Biotechnology Advances. 2009;27:195-226. DOI: 10.1016/j.biotechadv.2008.11.002

[64] Urrutia MM. General bacterial sorption processes. In: Wase J, Forster C, editors. Biosorbents for metal ions, CRC Press. UK: London; 1997. pp. 39-66

[65] Vasudevan P, Padmavathy V, Tewari N, Dhingra SC. Biosorption of heavy metal ions. Journal of Scientific and Industrial Research. 2001;60:112-120

[66] Kinoshita H, Sohma Y, Ohtake F, Ishida M, Kawai Y, Kitazawa H, Saito T, Kimura K. Biosorption of heavy metals by lactic acid bacteria and identification of mercury binding protein. Research in Microbiology. 2013;164(7):701-709. DOI: 10.1016/j.resmic.2013.04.004

[67] Vijayaraghavan K, Yun KS. Bacterial biosorbents and biosorption. Biotechnology Advances. 2008;26(3):266-291. DOI: 10.1016/j.biotechadv.2008.02.002

[68] Gourdon R, Bhende S, Rus E, Sofer SS. Comparison of cadmium biosorption by grampositive and gram-negative bacteria from activated sludge. Biotechnology Letters. 1990;12(11):839-842

[69] Das N, Vimala R, Karthika P. Biosorption of heavy metals - An overview. Indian Journal of Biotechnology. 2008;7:159-169

[70] Kuyicak N, Volesky B. Biosorption by fungal biomass. In: Volesky B, editor. Biosorption of Heavy Metals. Florida: CRC press; 1990. pp. 173-198

[71] Khani MH. Uranium biosorption by Padina sp. algae biomass: Kinetics and thermodynamics. Environmental Science and Pollution Research International. 2011;18:1593-1605

[72] Flouty R, Estephane G. Bioaccumulation and biosorption of copper and lead by a unicellular algae Chlamydomonas reinhardtii in single and binary metal systems: A comparative study. Journal of Environmental Management. 2012;111:106-114

[73] Trinelli MA, Areco MM, Mdos SA. Co-biosorption of copper and glyphosate by Ulva lactuca. Colloids and Surfaces B: Biointerfaces. 2013;105:251-258 
[74] Oyedepo TA. Biosorption of lead (II) and copper (II) metal ions on Calotropis procera (Ait.). Science Journal of Pure and Applied Chemistry. 2011;1:1-7

[75] Wang J, Chen C. Biosorption of heavy metals by saccharomyces cerevsiae: A review. Biotechnology Advances. 2006;24:427-451. DOI: 10.1016/j.biotechadv.2006.03.001

[76] Gadd GM, Griffiths AJ. Effect of copper on morphology of Aureobasidium pullulans. Transactions of the British Mycological Society. 1980;74:387-392

[77] Gadd GM, Mowll JL. Copper uptake by yeast-like cells, hyphae and chlamydospores of Aureobasidium pullulans. Experimental Mycology. 1985;9:230-240

[78] Remacle J. The cell wall and metal binding. In: Volesky B, editor. Biosorption of Heavy Metals. Boca Raton, Florida, USA: CRC Press; 1990. pp. 83-92

[79] Huang C, Huang CP. Application of Aspergillus oryzae and Rhizopus oryzae for Cu (II) removal. Water Research. 1996;30:1985-1990

[80] Kapoor A, Viraraghavan T. Fungi as biosorbent. In: DAJ W, Forster CF, editors. Biosorbents for Metal Ions. London, UK: Taylor and Francis; 1997. pp. 67-85

[81] Peregol P, Howell SB. Molecular mechanisms controlling sensitivity to toxic metal ions in yeast. Toxicology and Applied Pharmacology. 1997;147:312-318. DOI: 10.1006/ taap.1997.8271

[82] Eide D. Molecular biology of iron and zinc uptakes in eukaryotes. Current Opinion in Cell Biology. 1997;9:573-577

[83] Eide DJ. The molecular biology of metal ion transport in Saccharomyces cerevisiae. Annual Review of Nutrition. 1998;18:441-469. DOI: 10.1146/annurev.nutr.18.1.441

[84] Zhou. Microbiology. 2nd ed. Beijing: Higher Education Press; 2002. (in Chinese)

[85] Horikoshi T, Nakajima A, Sakaguchi R. Studies on the accumulation of heavy metal elements in biological systems: Accumulation of uranium by microorganisms. European Journal of Applied Microbiology and Biotechnology. 1981;12:90-96

[86] Veglio F, Beolchini F. Removal of metals by biosorption: A review. Hydrometallurgy. 1997;44:301-316

[87] Marques PA, Pinheiro HM, Teixeria JA, Rosa MF. Removal efficiency of $\mathrm{Cu}^{2+}, \mathrm{Cd}^{2+}$ and $\mathrm{Pb}^{2+}$ by waste brewery biomass: $\mathrm{pH}$ and cation association effects. Desalination. 1999; 124:137-144

[88] Park JK, Lee JW, Jung JY. Cadmium uptake capacity of two strains of Saccharomyces cerevisiae cells. Enzyme and Microbial Technology. 2003;33:371-378

[89] Bakkaloglu I, Butter TJ, Evison LM, Holland FS, Hancock IC. Screening of various types of biomass for removal and recovery of heavy metals $(\mathrm{Zn}, \mathrm{Cu}, \mathrm{Ni})$ by biosorption, sedminentation and desorption. Water Science and Technology. 1998;38:269-277 
[90] Soares EV, Coninck GD, Duarte F, Soares HMVM. Use of saccharomyces cerevisaie for $\mathrm{Cu} 2+$ removal from solution: The advantages of using a flocculent strain. Biotechnology Letters. 2002;24:663-666

[91] Mapolelo $M$, Torto N. Trace enrichment of metal ions in aquatic environments by Saccharomyces cerevisiae. Talanta. 2004;64:39-47. DOI: 10.1016/j.talanta.2003.10.058

[92] Kelly-Vargas K, Cerro-Lopez M, Reyna-Tellez S, Bandala ER, Sanchez-Salas JL. Biosorption of heavy metals in polluted water, using different waste fruit cortex. Physics and Chemistry of the Earth. 2012;37-39:26-29. DOI: 10.1016/j.pce.2011.03.006

[93] White C, Sayer JA, Gadd GM. Microbial solubilization and immobilization of toxic metals: Key biogeochemical processes for treatment of contamination. FEMS Microbiology Reviews. 1997;20(3-7):503-516

[94] Ahalya N, Ramachandra TV, Kanamadi RD. Biosorption of heavy metals. Research Journal of Chemistry and Environment. 2003;7:71-79

[95] Goyal N, Jain SC, Banerjee UC. Comparative studies on the microbial adsorption of heavy metals. Advances in Environmental Research. 2003;7:311-319

[96] Greene B, Darnall DW. Microbial oxygenic photoautotrophs (cyanobacteria and algae) for metal-ion binding. In: Ehrlich HL, Brierley CL, editors. Microbial Mineral Recovery. New York: McGraw-Hill; 1990. pp. 227-302

[97] Deng X, Wang P. Isolation of marine bacteria highly resistant to mercury and their bioaccumulation process. Bioresource Technology. 2012;121:342-347

[98] Gadd GM, White C. Copper uptake by Penicillium ochro-chloron: Influence of pH on toxicity and demonstration of energydependent copper influx using protoplasts. Journal of General Microbiology. 1985;131:1875-1879

[99] Modak JM, Natarajan KA. Biosorption of metals using nonliving biomass-a review. Minerals and Metallurgical Processing. 1995;12:189-196

[100] Gourdon R, Bhande S, Rus E, Sofer SS. Comparison of cadmium biosorption by grampositive and gram-negative bacteria from activated sludge. Biotechnology Letters. 1990;12(11):839-842

[101] Nuhoglu Y, Malkoc E. Investigations of nickel (II) removal from aqueous solutions using tea factory waste. Journal of Hazardous Materials. 2005;127:120. DOI: 10.1016/j. jhazmat.2005.06.030

[102] Zouboulis AL, Matis KA, Hancock IC. Biosorption of metals from dilute aqueous solutions. Separation and Purification Methods. 1997;26:255-295. DOI: 10.1080/0360 2549708014160

[103] Jalali-Rad R, Ghafourian H, Asef Y, Dalir ST, Sahafipour MH, Gharanjik BM. Biosorption of cesium by native and chemically modified biomass of marine algae: Introduce the new biosorbents for biotechnology applications. Journal of Hazardous Materials. 2004;116(1-2):125-134. DOI: 10.1016/j.jhazmat.2004.08.022 
[104] El-Sikaily A, El-Nemr A, Khaled A. Copper sorption onto dried red alga Pterocladia capillacea and its activated carbon. Chemical Engineering Journal. 2011;168(2):707-714. DOI: 10.1016/j.cej.2011.01.064

[105] Kiran I, Akar T, Tunali S. Biosorption of $\mathrm{Pb}$ (II) and $\mathrm{Cu}$ (II) from aqueous solution by pretreated biomass of Neurospora crassa. Process Biochemistry. 2005;40(11):3550-3558. DOI: $10.1016 /$ j.procbio.2005.03.051

[106] Alluri HK, Ronda SR, Settalluri VS, Bondili JS, Suryanarayana V, Venkateshwar P. Biosorption: An eco-friendly alternative for heavy metal removal. African Journal of Biotechnology. 2007;6:2924-2931

[107] Ahemad M, Kibret M. Recent trends in microbial biosorption of heavy metals: A review. Biochemistry and Molecular Biology. 2013;1(1):19-26

[108] Dey U, Chatterjee S, Mondal NK. Isolation and characterization of arsenic-resistant bacteria and possible application in bioremediation. Biotechnology Reports. 2016;10:1-7. DOI: 10.1016/j.btre.2016.02.002

[109] Banerjee S, Datta S, Chattyopadhyay S, Sarkar P. Arsenic accumulation and transforming bacteria from contaminated soil for potential use in bioremediation. Journal of Environmental Science and Health. Part A. Toxic/Hazardous Substances and Environmental Engineering. 2011;46(14):1736-1747. DOI: 10.1080/10934529.2011. 623995

[110] Majumder A, Ghosh S, Saha N, Kole SC, Sarkar S. Arsenic accumulating bacteria isolated from soil for possible application in bioremediation. Journal of Environmental Biology. 2013;34(5):841-846

[111] Shamim S, Rehman A. Physicochemical surface properties of Cupriavidus metallidurans CH34 and Pseudomonas putida $\mathrm{mt} 2$ under cadmium stress. Journal of Basic Microbiology. 2013;54(4):306-314. DOI: 10.1002/jobm.201200434

[112] Shamim S, Rehman A, Qazi MH. Swimming, swarming, twitching and chemotactic response of Cupriavidus metallidurans $\mathrm{CH} 34$ and Pseudomonas putida $\mathrm{mt} 2$ in the presence of cadmium. Archives of Environmental Contamination and Toxicology. 2013;66(3):407414. DOI: $10.1007 / \mathrm{s} 00244-013-9966-5$

[113] Shamim S, Rehman A, Qazi MH. Cadmium resistance mechanism of Cupriavidus metallidurans CH34 and Pseudomonas putida $\mathrm{mt} 2$. Archives of Environmental Contamination and Toxicology. 2013;67(2):149-157. DOI: 10.1007/s00244-014-0009-7

[114] Shamim S, Rehman A. Antioxidative enzyme profiling and biosorption ability of Cupriavidus metallidurans $\mathrm{CH} 34$ and Pseudomonas putida $\mathrm{mt} 2$ under cadmium stress. Special issue, microbes in bioremediation. Journal of Basic Microbiology. 2015;55(3):374-381. DOI: 10.1002/jobm.201300038 
[115] Rani MJ, Hemambika B, Hemapriya J, Kannan VR. Comparative assessment of heavy metal removal by immobilized and dead bacterial cells: A biosorption approach. African Journal of Environmental Science and Technology. 2010;4(2):077-083

[116] Congeevaram S, Dhanarani S, Park J, Dexilin M, Thamaraiselvi K. Biosorption of chromium and nickel by heavy metal resistant fungal and bacterial isolates. Journal of Hazardous Materials. 2007;146:270-277. DOI: 10.1016/j.jhazmat.2006.12.017

[117] Sulaymon AH, Mohammed AA, Al-Musawi TJ. Competitive biosorption of lead, cadmium, copper, and arsenic ions using algae. Environmental Science and Pollution Research International. 2012;20:3011-3023. DOI: 10.1007/s11356-012-1208-2

[118] Jencarova J, Luptakova A. The elimination of heavy metal ions from waters by biogenic iron sulphides. Chemical Engineering Transactions. 2012;28:205-210

[119] Babak L, Šupinova P, Zichova M, Burdychova R, Vitova E. Biosorption of Cu, Zn and $\mathrm{Pb}$ by thermophilic bacteria - Effect of biomass concentration on biosorption capacity. Acta Universitatis Agriculturae Et Silviculturae Mendelianae Brunesis. 2012;60:9-18

[120] Gao R, Wang Y, Zhang Y, Dai W. Cobalt (II) bioaccumulation and distribution in Rhodopsseudomonas palustris. Biotechnology and Biotechnological Equipment. 2017;31(3): 527-537. DOI: 10.1080/13102818.2017. 1292148

[121] Karakagh RM, Chorom M, Motamedi H, Kalkhajeh YKY, Oustan S. Biosorption of Cd and $\mathrm{Ni}$ by inactivated bacteria isolated from agricultural soil treated with sewage sludge. Ecohydrology and Hydrobiology. 2012;12(3):191-198. DOI: 10.1016/S1642-3593(12) 70203-3

[122] Samarth DP, Chandekar CJ, Bhadekar RK. Biosorption of heavy metals from aqueous solution using Bacillus licheniformis. International Journal of Pure and Applied Sciences and Technology. 2012;10(2):12-19

[123] NagashettiV, Mahadevaraju GK, MuralidharTS, Javed A, TrivediD, Bhusal KP. Biosorption of heavy metals from soil by Pseudomonas aeruginosa. International Journal of Innovative Technology and Exploring Engineering (IJITEE). 2013;2(6):9-17

[124] Hussein H, Ibrahim SF, Kandeel K, Moawad H. Biosorption of heavy metals from waste water using Pseudomonas sp. Electronic Journal of Biotechnology. 2004;7(1):1-2. DOI: 10.2225/vol7-issue1-fulltext-2

[125] Monsieurs P, Hobman J, Vandenbussche, Mergeay M, Houdt RV. Response of Cupriavidus metallidurans CH34 to metals. In: Mergeay M, Houdt RV, editors. Metal Response in Cupriavidus Metallidurans; Volume I: From Habitats from Genes and Proteins. Springer Briefs in Molecular Science, Biometals. pp. 45-89

[126] Hemambika B, Rani MJ, Kannan VR. Biosorption of heavy metals by immobilized and dead fungal cells: A comparative assessment. Journal of Ecology and the Natural Environment. 2011;3(5):168-175 
[127] Romera E, Gonzalez F, Ballester A, Blazquez ML, Munoz JA. Biosorption with algae: A statistical review. Critical Reviews in Biotechnology. 2006;26:223-235. DOI: 10.1080/ 07388550600972153

[128] Viraraghavan T, Srinivasan A. Fungal biosorption and biosorbents. In: Kotrba P, Mackova M, Macek T, editors. Microbial Biosorption of Metals. Dordrecht: Springer; 2011. pp. 143158. DOI: 10.1007/978-94-007-0443-5-6

[129] Gina KY, Tanga YZ, Aziz MA. Derivation and application of a new model for heavy metal biosorption by algae. Water Research. 2002;36:1313-1323

[130] Sheikha D, Ashour I, Abu Al-Rub FA. Biosorption of zinc on immobilized green algae: Equilibrium and dynamics studies. The Journal of Engineering Research. 2008;5(1): 20-29

[131] Ramsenthil R, Meyyappan R. Single and multi-component biosorption of copper and zinc ions using microalgal resin. International Journal of Environmental Science and Development. 2010;1(4):298-301

[132] Subhashini S, Kaliappan S, Velan M. Removal of heavy metal from aqueous solution using Schizosaccharomyces pombe in free and alginate immobilized cells. In: 2nd International Conference on Environmental Science and Technology IPCBEE. Vol. 6. Singapore: IACSIT Press; 2011. pp. V2107-V2111

[133] Hajar M. Biosorption of cadmium from aqueous solution using dead biomass of brown alga Sargassum sp. Chemical Engineering Transactions. 2009;17:1173-1178. DOI:10.3303/ CET0917196

[134] Brinza L, Dring MJ, Gavrilescu M. Marine micro and macro algal species as biosorbents for heavy metals. Environmental Engineering and Management Journal. 2007;6: 237-251

[135] Kumar JI, Oommen C. Removal of heavy metals by biosorption using freshwater alga Spirogyra hyaline. Journal of Environmental Biology. 2012;33(1):27-31

[136] Yavuz H, Denizli A, Güngünes H, Safarikova M, Safarik I. Biosorption of mercury on magnetically modified yeast cells. Separation and Purification Technology. 2006;52:253260. DOI: 10.1016/j.seppur.2006.05.001

[137] Lodeiro P, Cordero B, Barriada JL, Herrero R, de Vicente MES. Biosorption of cadmium by biomass of brown marine macroalgae. Bioresource Technology. 2002;96(16):1796-1803. DOI: 10.1016/j.biortech.2005.01.002

[138] Brahmbhatt, Rinku NH, Patel V, Jasrai RT. Removal of cadmium, chromium and lead from filamentous alga of Pithophora sp. of industrial wastewater. International Journal of Environmental Sciences. 2012;3(1):408-411. DOI: 10.6088/ijes.2012030131039 
[139] Saravanan, Brindha V, Krishnan S. Studies on the structural changes of the biomass Sargassum sp. Journal of Advanced Bioinformatics Applications and Research. 2011; 2(3):193-196

[140] Mamisahebei S, Khaniki GRJ, Torabian A, Nasseri S, Naddafi K. Removal of arsenic from an aqueous solution by pretreated waste tea fungal biomass. Journal of Environmental Health Science and Engineering. 2007;4(2):85-92

[141] Rodríguez IA, Martínez-Juárez VM, Cárdenas-González JF, Moctezume-Zárate. Biosorption of arsenic (III) from aqueous solutions by modified fungal biomass of Paecilomyces sp. Bioiorganic Chemistry and Applications. 2013;2013:1-5. DOI: 10.1155/ 2013/376780

[142] Martínez-Júarez VM, Cárdenas-González JF, Torre-Bouscoulet ME, Acosta-Rodríguez I. Biosorption of mercury (II) from aqueous solutions onto fungal biomass. Bioinorganic Chemistry and Applications. 2012;2012:1-5. DOI: 10.1155/2012/156190

[143] Bunluesin S, Kruatrachue M, Pokethitiyook P, Upathan S, Lanza GR. Batch and continuous packed column studies of cadmium biosorption by Hydrilla verticillata biomass. Journal of Bioscience and Bioengineering. 2007;103(6):509-513. DOI: 10.1263/jbb.103.509

[144] Kujan P, Prell A, Safar H, Sobotka M, Rezanka T, Holler P. Use of the industrial yeast Candida utilis for cadmium sorption. Folia Microbiology (Praha). 2006;54(4):257-260

[145] Javaid A, Bajwa R. Biosorption of Cr (III) ions from tannery wastewater by Pleurotus ostreatus. Mycopathologia. 2007;5:71-79

[146] Park D, Yun Y-S, Park JM. Use of dead fungal biomass for the detoxification of hexavalent chromium: Screen and kinetics. Process Biochemistry. 2005;40:2559-2565

[147] Cossich ES, Tavares CRG, Ravagnani TMK. Biosorption of chromium (III) by Sargassum sp. biomass. Electronic Journal of Biotechnology. 2002;5(2):133-140

[148] El-Kassas HY, El-Taher EM. Optimization of batch process parameters by response surface methodology for mycoremediation of chrome-VI by a chromium resistant strain of marine Trichoderma viride. American-Eurasian Journal of Agriculture and Environmental Science. 2009;5(5):676-681

[149] Sethi BK, Kanungo S, Rout JR, Nanda PK, Sahoo SL. Effect of chromium on Mucor species and optimization of growth conditions. Nature and Science. 2010;8(4):29-32

[150] Jha S, Dikshit SN, Pandey G. Comparative study of growing/immobilized biomass verses resting biomass of $A$. lentulus for the effect of $\mathrm{pH}$ on $\mathrm{Cu}^{2+}$ metal removal. Research Journal of Pharmaceutical, Biological and Chemical Sciences. 2012;3(3):421-427

[151] Parungao MM, Tacata PS, Tanayan CRG, Trinidad LC. Biosorption of copper, cadmium and lead by copper-resistant bacteria isolated from Mogpog River, Marinduque. The Philippine Journal of Science. 2007;136(2):155-165 
[152] Sutherland C, Venkobachar C. A diffusion-chemisorption kinetic model for simulating biosorption using forest macro-fungus, fomes fasciatus. International Research Journal of Plant Science. 2010;1(4):107-117

[153] Hassan SW, El-Kassas HY. Biosorption of cadmium from aqueous solutions using a local fungus Aspergillus cristatus (Glaucus group). African Journal of Biotechnology. 2012;11(9):2276-2286. DOI: 10.5897/AJB11.3140

[154] Osman MS, Bandyopadhyay M. Biosorption of lead (II) ions from wastewater by using a fungus P. ostreatus. Journal of Civil Engineering. 1999;27(2):193-196

[155] Barros LM Jr, Macedo GR, Duarte MML, Silva EP, Lobato AKCL. Biosorption of cadmium using the fungus Aspergillus niger. Brazilian Journal of Chemical Engineering. 2003;20(3):229-239. DOI: 10.1590/S0104-66322003000300003

[156] Murugesan GS, Sathishkumar M, Swaminathan K. Arsenic removal from groundwater by pretreated waste tea fungal biomass. Bioresource Technology. 2006;97(3):483-487. DOI: 10.1016/j.biortech.2005.03.008

[157] Tay C-C, Liew H-H, Yong S-K, Surif S, Redzwan G, Abdul-Talib S. Cu (II) removal onto fungal derived biosorbents: Biosorption performance and the half saturation constant concentration approach. International Journal of Research in Chemistry and Environment. 2012;2(3):138-143

[158] Purkayastha RP, Mitra AK. Metal uptake by mycelia during submerged growth and by sporocarps of an edible fungus Volvariella volvacea. Indian Journal of Experimental Biology. 1992;30:1184-1187

[159] Muraleedharan TR, Venobachar C. Mechanism of biosorption of copper (II) by Ganoderma lucidium. Biotechnology and Bioengineering. 1990;35:320-325

[160] Muraleedharan TR, Iyengar L, Venkobachar C. Screening of tropical wood rotting mushrooms for copper biosorption. Applied and Environmental Microbiology. 1995;61: 3507-3508

[161] García MA, Alonso J, Melgar MJ. Agaricus macrosporus as a potential bioremediation agent for substrates contaminated with heavy metals. Journal of Chemical Technology and Biotechnology. 2005;80:325-330. DOI: 10.1002/jctb.1203

[162] Anaemene IA. The use of Candida sp. in the biosorption of heavy metals from industrial effluent. European Journal of Experimental Biology. 2012;2(3):484-488

[163] Arakaki AH, Vandenberghe LPS, Soccol VT, Musakai R, FilhoEFD, GregórioA, SoccolCR. Optimization of biomass production with copper bioaccumulation by yeasts in submerged fermentation. Brazilian Archives of Biology and Technology. 2011;54(5):10271034. DOI: $10.1590 /$ S1516-89132011000500021

[164] Apinthanapong M, Phensaijai M. Biosorption of copper by spent yeast immobilized in sodium alginate beads. Kasetsart Journal (Natural Science). 2009;43:326-332 
[165] Prakash BS, Kumar SV. Batch removal of heavy metals by biosorption onto marine algae-equilibrium and kinetic studies. International Journal of Chemical Technology Research. 2013;5(3):1254-1262

[166] Muraleedharan TR, Iyengar L, Venkobachar C. Biosorption: An attractive alternative for metal removal and recovery. Current Science. 1991;61(6):379-385 
